\title{
Composition and fate of short-period super-Earths
}

\section{The case of CoRoT-7b}

\author{
D. Valencia ${ }^{1}$, M. Ikoma ${ }^{1,2}$, T. Guillot ${ }^{1}$, and N. Nettelmann ${ }^{3}$ \\ 1 Observatoire de la Côte d'Azur, Université de Nice-Sophia Antipolis, CNRS UMR 6202, BP 4229, 06304 Nice Cedex 4, France \\ e-mail: valencia@oca.eu \\ 2 Dept. of Earth and Planetary Sciences, Tokyo Institute of Technology, Ookayama, Meguro-ku, Tokyo 152-8551, Japan \\ 3 Institut für Physik, Universität Rostock, 18051 Rostock, Germany
}

Received 7 July 2009 / Accepted 7 February 2010

\section{ABSTRACT}

\begin{abstract}
Context. The discovery of CoRoT-7b, a planet of a radius $1.68 \pm 0.09 R_{\oplus}$, a mass $4.8 \pm 0.8 M_{\oplus}$, and an orbital period of 0.854 days demonstrates that small planets can orbit extremely close to their star.

Aims. Several questions arise concerning this planet, in particular concerning its possible composition, and fate.

Methods. We use knowledge of hot Jupiters, mass loss estimates and models for the interior structure and evolution of planets to understand its composition, structure and evolution.

Results. The inferred mass and radius of CoRoT-7b are consistent with a rocky planet that would be significantly depleted in iron relative to the Earth. However, a one sigma increase in mass $\left(5.6 M_{\oplus}\right)$ and one sigma decrease in size $\left(1.59 R_{\oplus}\right)$ would make the planet compatible with an Earth-like composition (33\% iron, 67\% silicates). Alternatively, it is possible that CoRoT-7b contains a significant amount of volatiles. For a planet made of an Earth-like interior and an outer volatile-rich vapour envelope, an equally good fit to the measured mass and radius is found for a mass of the vapour envelope equal to 3\% (and up to 10\% at most) of the planetary mass. Because of its intense irradiation and small size, we determine that the planet cannot possess an envelope of hydrogen and helium of more than $1 / 10000$ of its total mass. We show that a relatively significant mass loss $\sim 10^{11} \mathrm{~g} \mathrm{~s}^{-1}$ is to be expected and that it should prevail independently of the planet's composition. This is because to first order, the hydrodynamical escape rate is independent of the mean molecular mass of the atmosphere, and because given the intense irradiation, even a bare rocky planet would be expected to possess an equilibrium vapour atmosphere thick enough to capture stellar UV photons. Clearly, this escape rate rules out the possibility that a hydrogen-helium envelope is present, as it would escape in only $\sim 1 \mathrm{Ma}$. A water vapour atmosphere would escape in $\sim 1 \mathrm{Ga}$, indicating that this is a plausible scenario. The origin of CoRoT-7b cannot be inferred from the present observations: It may have always had a rocky composition; it may be the remnant of a Uranus-like ice giant, or a gas giant with a small core that has been stripped of its gaseous envelope.

Conclusions. With high enough sensitivity, spectroscopic transit observations of CoRoT-7 should constrain the composition of the evaporating flow and therefore allow us to distinguish between a rocky planet and a volatile-rich vapour planet. In addition, the theoretical tools developed in this study are applicable to any short-period transiting super-Earth and will be important to understanding their origins.
\end{abstract}

Key words. planets and satellites: individual: CoRoT-7b

\section{Introduction}

The newest planet discovered by space mission CoRoT is remarkably interesting. CoRoT-7b is not only the first super-Earth with a measured radius, but orbits extremely close to its parent star, only 4.27 stellar radii away (Leger et al. 2009). Its radius and orbital period are $R=1.68 \pm 0.09 R_{\oplus}$, and $P=0.854$ days respectively, the calculated age and equilibrium temperature are 1.2-2.3 Ga and 1800-2600 K (Leger et al. 2009) respectively, and the mass reported by radial velocity measurements is $M=$ $4.8 \pm 0.8 M_{\oplus}$ (Queloz et al. 2009).

While the combination of mass $(M)$ and radius $(R)$ measurements alone does not yield a unique solution for the composition of a planet (Valencia et al. 2007b; Adams et al. 2008), the short period of CoRoT-7b and consequently the strong irradiation on the planet, may help constrain its composition. We use structure models and atmospheric evaporation scenarios to investigate the physical nature and possible origin of CoRoT-7b. We start by considering the fate of an atmosphere (Sect. 2) before turning to the planet's structure. Despite the intrinsic problem of degeneracy in composition, we can establish if a planet is too large to be only rocky (its radius is larger than the maximum size of a coreless magnesium-silicate planet), or even too large to be icy (its radius is larger than the maximum size of a snowball planet) given its mass. We describe the model used to calculate the planet's structure (Sect. 3) and show that for a subset of radius-mass combinations within the data, CoRoT-7b would actually be too large to be composed of only refractory material. We present our results on the composition of CoRoT-7b and discuss possible evolution scenarios, including a case for the planet as an evaporated ice or gas giant (Sect. 4). We conclude by providing arguments for the most likely scenario for CoRoT-7b.

The framework presented here is applicable to any transiting close-in super-Earth. Moreover, owing to the bias of discovering short-period planets, we expect many such super-Earths to be discovered in the near future with the next phases of CoRoT and Kepler's observations.

\section{Mass loss}

Close-in planets are vulnerable to evaporation because of intense irradiation from their parent star. Indeed, gas has been 
detected to flow out from the transiting gas giant HD 209458b (Vidal-Madjar et al. 2003). Certainly, CoRoT-7b, whose mean density is $4-8 \mathrm{~g} \mathrm{~cm}^{-3}$, is denser than HD 209458b $(\bar{\rho}=$ $0.33 \mathrm{~g} \mathrm{~cm}^{-3}$ ) by at least one order of magnitude. However, the UV flux received by CoRoT-7b is greater by one order of magnitude, because it is closer to its parent star and somewhat younger than HD 209458b (0.017 AU and 1.2-2.3 Ga compared to $0.047 \mathrm{AU}$ and $\sim 4 \mathrm{Ga}$ ). One could therefore expect mass loss rates that are comparable within an order of magnitude for the two planets. Consequently, given that CoRoT-7b is 40 to 55 times less massive than HD 209458b, this mass loss may have a profound effect on its evolution, fate and present composition. We now attempt to quantify this mass loss, using simple assumptions (the precise modelling of atmospheric escape in this planet is a difficult task, which is beyond the scope of this article).

We model the atmospheric escape with the well-known expression for the extreme case of energy-limited escape, the validity of which has been verified for gas-giant planets close to their star (see review by Yelle et al. 2008):

$\dot{M}_{\mathrm{esc}}=\frac{3 \epsilon F_{\mathrm{EUV}}}{4 G \bar{\rho}_{\mathrm{p}} K_{\mathrm{tide}}}$,

where $F_{\mathrm{EUV}}$ is the incident flux of the stellar EUV (extreme ultraviolet radiation), $\epsilon$ is the heating efficiency defined as the ratio of the net heating rate to the rate of stellar energy absorption, $\bar{\rho}_{\mathrm{p}}$ is the mean density of the planet, $K_{\text {tide }}$ is a correction factor to include the Roche-lobe effect (Erkaev et al. 2007; Lecavelier des Etangs et al. 2004), and $G$ is Newton's constant. Of course, most of the physics is hidden in the parameter $\epsilon$, which is mainly controlled by the ability of the upper atmosphere to cool. For close-in gas giants, detailed calculations show that $\mathrm{H}_{3}^{+}$plays a dominant role for the cooling of the upper atmosphere. In particular, Eq. (1) with $\epsilon=0.4$ and HD 209458b's characteristic values yields $\dot{M}_{\text {esc }}=4 \times 10^{10} \mathrm{~g} \mathrm{~s}^{-1}$, which is to be compared to values between 3.5 and $4.8 \times 10^{10} \mathrm{~g} \mathrm{~s}^{-1}$ obtained in the literature (Yelle et al. 2008, and references therein).

For planets with different atmospheric compositions, one may question the validity of the relation. Tian et al. (2008) have recently simulated the escape of the Earth's atmosphere for different EUV irradiation levels. They demonstrate that for EUV irradiation fluxes above $\sim 10$ times the solar value, the atmosphere is in the hydrodynamic regime, namely that it escapes in an energy-limited fashion rather than through blow-off or in a diffusion-limited way. While thermal conduction is important for moderate EUV fluxes, implying that mass loss then depends on atmospheric composition, its contribution is found to become negligible for strong EUV fluxes - as it is in our case -. This can also be seen by the fact that the ratio of the EUV flux that the planet receives to a typical energy flux due to thermal conduction is $\beta \approx 10^{10-11}$, and that the ratio of a typical energy flux to a thermal conduction flux is $\zeta \approx 10^{3-5}$, provided that the thermal conduction coefficient is of the same order of magnitude as that for hydrogen molecules (see García Muñoz 2007, for a similar discussion about HD 209458 b; and Watson et al. 1981, for a precise definition of $\beta$ and $\zeta$ ).

As described above, the escape efficiency is controlled by the radiative cooling by $\mathrm{H}_{3}^{+}$for hydrogen atmospheres. In the case of water-rich atmospheres, oxygen from dissociation of $\mathrm{H}_{2} \mathrm{O}$ prevents a significant amount of $\mathrm{H}_{3}^{+}$from forming, which means the efficiency might be higher (e.g., García Muñoz 2007).

Indeed, for Earth's exobase temperature and velocity values, the corresponding mass loss rate as suggested by Fig. 8 in Tian et al. (2008) is of the order of $10^{9} \mathrm{~g} / \mathrm{s}$ for the highest EUV flux $\left(=100 \mathrm{erg} \mathrm{s}^{-1} \mathrm{~cm}^{-2}\right)$. This is to be compared with a value of $\dot{M}_{\text {esc }} \sim 10^{9} \epsilon \mathrm{g} / \mathrm{s}$ obtained with the use of Eq. (1) under the same conditions.

For silicate atmospheres, no calculations exist, but we can presume that in the likely absence of species that cool much more efficiently than $\mathrm{H}_{3}^{+}$, Eq. (1) with $\epsilon=0.4$ should remain valid within an order of magnitude -we will come back to this particular case in Sect. 4.1.3. In conclusion, mass loss should remain substantial whatever the properties of the atmosphere (and its mean molecular weight).

Another important quantity controlling the escape flux is the flux of EUV photons emitted by the star, which is strongly dependent on the stellar age

$F_{\mathrm{EUV}}=\alpha t_{9}^{-\beta} a_{1}^{-2}$

according to recent observations of EUV emission from young stars (Ribas et al. 2005); where $t_{9}$ is the stellar age in Ga, $a_{1}$ the planet's orbital distance in $\mathrm{AU}$, and $\alpha$ and $\beta$ are constants. Their best-fit result for Sun-like stars similar to CoRoT-7 is obtained with $\alpha=29.7 \mathrm{erg} \mathrm{s}^{-1} \mathrm{~cm}^{-2}\left(\equiv \alpha_{\mathrm{R} 05}\right)$ and $\beta=1.23$. With this expression and these values, one obtains $F_{\mathrm{EUV}}=$ $5.0 \times 10^{4} \mathrm{erg} \mathrm{s}^{-1} \mathrm{~cm}^{-2}$ for CoRoT-7b $\left(t_{9}=1.8\right.$ and $\left.a_{1}=0.017\right)$, while $2.4 \times 10^{3} \mathrm{erg} \mathrm{s}^{-1} \mathrm{~cm}^{-2}$ for HD209458 b $\left(t_{9}=4.0\right.$ and $\left.a_{1}=0.047\right)$.

We note that with such a high UV irradiation flux, CoRoT-7b may be above the purely energy-limited escape regime and in a regime limited by the recombination of electrons and hydrogen nuclei, implying $\dot{M}_{\text {esc }} \propto\left(F_{\text {EUV }}\right)^{0.6}$ (Murray-Clay et al. 2009). This would imply mass loss rates about twice lower than estimated here with $\epsilon=0.4$ and Eq. (1). As we are concerned with orders of magnitude estimates, this possibility will be ignored in the rest of the work.

With values characteristic of CoRoT-7b in Eq. (1), one obtains

$\dot{M}_{\mathrm{esc}}=1 \times 10^{11} t_{9}^{-\beta} f_{\mathrm{esc}} \mathrm{g} \mathrm{s}^{-1}$,

with

$f_{\mathrm{esc}}=\left(\frac{\alpha}{\alpha_{\mathrm{R} 05}}\right)\left(\frac{a_{1}}{0.017}\right)^{-2}\left(\frac{\epsilon}{0.4}\right)\left(\frac{\bar{\rho}_{p}}{\bar{\rho}_{\oplus}}\right)^{-1}\left(\frac{K_{\mathrm{tide}}}{0.65}\right)^{-1}$

With the reported age of CoRoT-7b, $t_{9}=1.2-2.3$ (Leger et al. 2009 ), and $\beta=1.23$, Eq. (3) yields values for the escape rate, $\dot{M}_{\mathrm{esc}}=(5-10) \times 10^{10} \mathrm{~g} \mathrm{~s}^{-1}$, similar to that for HD $209458 \mathrm{~b}$.

To obtain the total mass lost before $t_{9}$, we integrate Eq. (1) so that

$M_{\mathrm{esc}}=0.7\left(\frac{t_{9}^{1-\beta}-t_{9,0}^{1-\beta}}{1-\beta}+t_{9,0}^{1-\beta}\right) f_{\mathrm{esc}} M_{\oplus}$

where $t_{9,0}$ is the time during which the EUV flux is constant and taken to be 0.1. Using Eq. (5) with $\epsilon=0.4$, assuming a planetary density that is constant in time and CoRoT-7b's characteristic values, one obtains a cumulative escaped mass that goes from $\sim 2.3 M_{\oplus}$ for a $5.8 M_{\oplus}$ planet to $\sim 4.5 M_{\oplus}$ for a $4 M_{\oplus}$ planet. Hence, CoRoT-7b's present mass agrees with a planet that has not lost much more than half its initial mass. However, this picture can be significantly modified when the planet's density varies greatly with time, namely, in the case of planets with vapour or hydrogen envelopes, as we will see in Sect. 4.2.5.

Without a detailed calculation of heating and cooling effects, which depend on the exact composition of the escaping atmosphere, this should be considered only as an order of magnitude estimate. But it shows that for any atmosphere to be present, it 
must constantly be resupplied and that the planet may have already lost a significant fraction of its mass. On the other hand, this does not mean that this planet happened to be detected on its way to complete evaporation. By integrating Eq. (3), we find that the current state is rather stable, mainly because of the weakened EUV; complete evaporation takes more than $10 \mathrm{Ga}$ for $\epsilon=0.4$.

In any case, our estimates leave room for a plethora of possibilities concerning the global composition of the planet: it may possess iron and rocks, but also volatiles or even hydrogen and helium, and the question of how much of these may be present arises. We attempt to address this in the sections below.

\section{Modelling interior structure and evolution}

\subsection{Procedure}

In order to calculate the possible structure and evolution of Earth-like planets up to ice giants and gas giants, we combined two models. For the solid/liquid regions we used a three layer (iron/rock/ice) hydrostatic model based on Vinet and shock equations of states; each layer is assumed to be isentropic except for the conductive thermal boundary layers at the top and bottom of the mantle (Valencia et al. 2006, 2007a). This model reproduces the Earth's structure well and has been used previously to understand super-Earths properties. For gaseous/fluid envelopes, we used a quasi-static model of interior structure and evolution that has been extensively used to model solar and extrasolar giant planets (Guillot \& Morel 1995; Guillot 2005). The two models are tied by using the pressure at the bottom of the gaseous/fluid envelope as an upper boundary condition for the calculation of the structure of the solid/liquid interior. The temperature was not consistently calculated between the two models. However, this should not affect the results, because thermal effects have a negligible impact on the properties of high-pressure iron, rocks and solid ices.

Our purpose is to understand possible compositions of CoRoT-7b. The thermal evolution of such a planet is uncertain, because it depends on its composition, initial state, and dynamical evolution, all of which are unknown. It also depends on atmospheric properties and opacities, two quantities that are difficult to estimate for a planet that probably has a very different atmospheric composition from what was usually considered. Fortunately, those two quantities have only a small impact on our results, as described below. Following Guillot (2005), evolution calculations were obtained using a simplified atmospheric boundary condition

$T_{10}=T_{0 *}\left(1+L / L_{\mathrm{eq}}\right)^{1 / 4}$,

where $T_{10}$ is the temperature at the 10 bar level, $L$ is the planet's intrinsic luminosity, $L_{\mathrm{eq}}$ corresponds to the stellar luminosity that it receives and $T_{0 *}$ is chosen equal to $2500 \mathrm{~K}$ to account for the presence of a greenhouse effect similar to what is obtained for the atmospheres of close-in giant exoplanets (e.g. Iro et al. 2005). Indeed, because the thermal evolution of highly irradiated planets is rapidly governed by the growth of an inner radiative zone, it is weakly dependent on the choice of the outer boundary condition. What is most important to us is that the high $(\sim 2000 \mathrm{~K})$ irradiation temperatures of CoRoT-7b maintain the atmosphere well above the condensation temperature of water, so that a vapour atmosphere may be present for a long time. (This is contrary to planets in colder environments, which require a large $L$ to maintain photospheric water vapour, and therefore cool quickly until they become solid.)
For opacities in gaseous envelopes, we used the Rosseland opacity table of Alexander \& Ferguson (1994). The table is valid for a hydrogen-helium solar composition mixture, so that its application to other atmospheres (e.g. one mainly formed with water vapour) may be questioned. We point out however that the cooling is generally controlled by the opacity in a region at a pressure $P \sim 1-10 \mathrm{kbar}$ and $T \sim 3000 \mathrm{~K}$, for which the opacities are extremely uncertain, regardless of the assumed composition (Guillot et al. 1994). At these pressures and temperatures, it is mostly controlled by collision-induced absorption by molecules in the infrared, and by the presence of electrons that yield important absorption (e.g. from $\mathrm{H}^{-}$for a hydrogen rich gas) at visible wavelengths. As a result, the opacities increase rapidly with increasing $P$ and $T$, whatever the assumed composition. The switch from an almost isothermal external layer to a nearly adiabatic envelope in deeper regions is expected to occur abruptly. In this case also the quantitative uncertainties on the underlying physical parameters are large, but they have a limited impact on the result, and they do not qualitatively change our conclusions.

Finally, the boundary condition at the bottom of the envelope is defined as a radius provided by the hydrostatic model of the solid/liquid interior, and a luminosity

$L_{0}=\dot{\epsilon}_{\text {radioactive }} M_{\mathrm{R}}+C_{V} \frac{\mathrm{d} T_{\mathrm{Fe}+\mathrm{R}}}{\mathrm{d} t} M_{\mathrm{Fe}+\mathrm{R}}$,

where $\dot{\epsilon}_{\text {radioactive }}$ is the radioactive luminosity per unit mass, $M_{\mathrm{R}}$ is the mass of the (rocky) mantle, $C_{V}$ is the rock + iron core specific heat, and $T_{\mathrm{Fe}+\mathrm{R}}$ is a characteristic temperature of the solid region of mass $M_{\mathrm{Fe}+\mathrm{R}}$. In the calculations we assumed a chondritic value $\dot{\epsilon}_{\text {radioactive }}=2 \times 10^{20} \mathrm{erg} \mathrm{s}^{-1} M_{\oplus}^{-1}, C_{V}=$ $10^{7} \mathrm{erg} \mathrm{g}^{-1} \mathrm{~K}^{-1}$ and $\mathrm{d} T_{\mathrm{Fe}+\mathrm{R}} / \mathrm{d} t=\mathrm{d} T_{\text {env }} / \mathrm{d} t$, where $T_{\text {env }}$ is the temperature at the bottom of the envelope. However, we found our results to be quite insensitive to the choice of the inner boundary condition, because for most cases, $L$ was found to be significantly higher than $L_{0}$.

\subsection{States of matter inside CoRoT-7b}

We now describe the different phases and states of matter for a generic super-Earth given all possible compositions and emphasize the relevant structure for a short-period planet like CoRot-7b.

\subsubsection{Hydrogen and helium}

In Uranus and Neptune, hydrogen and helium form about 1 to $4 M_{\oplus}$ of the planets' outer envelopes (e.g Guillot 2005, and references therein). While it is not necessarily expected in a planet as small as CoRoT-7b, it is interesting to consider them and provide upper limits to their abundances in the planet.

Of course, given the temperatures to be considered ( $2000 \mathrm{~K}$ and above) and pressures well below a Mbar, hydrogen and helium are expected to behave as a gas with hydrogen in molecular form (see phase diagram in Guillot 2005). The equation of state considered for modelling their behaviour is that of Saumon et al. (1995).

\subsubsection{Water and "volatiles"}

Because of their high abundances, moderately refractory species like water, methane, and ammonia are crucial building blocks of planetary systems. They are often grouped within the 


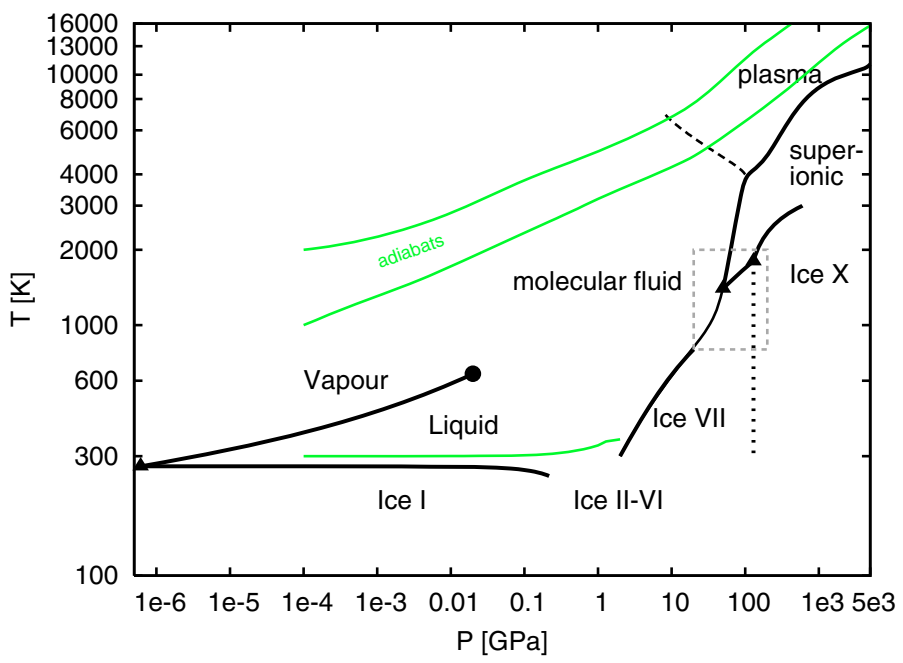

Fig. 1. Phase diagram of water from 0.01 bar to 50 Mbar and 300 to 16000 K. Black solid lines: phase-transition boundaries, Triangles: triple points, Circle: critical point; grey solid lines: adiabats starting from 300, 1000, and $2000 \mathrm{~K}$ respectively at 1 bar; black dashed line: continuous transition from molecular dissociated water to water plasma; grey dashed box: region of high uncertainty and contradictive experimental and theoretical results. For $P>4 \mathrm{GPa}$ and $T \geq 1000$ this diagram relies on data from FT-DFT-MD calculations French et al. (2009). The melting curve is taken from Feistel et al. (2006), the saturation curve from Wagner \& Pruß (2002), and the phase boundary of Ice VII is adapted from Goncharov et al. (2009) for pressures lower than $30 \mathrm{GPa}$. For higher pressures, experimental and theoretical investigations predict different phase boundaries Ice VII-molecular water, Ice VII-superionic water, Ice VII-Ice-X (controversial as a hard boundary from experiments, Hemley et al. 1987), IceX-superionic water and different locations of corresponding triple points French et al. (2009); Goncharov et al. (2009); Schwager et al. (2004).

denomination of "ices" in the literature. In order to avoid the confusion with solid water, we prefer to call them "volatiles" and will use this term throughout the rest of the article. In a primordial disc with near-solar composition and temperatures below $\sim 200 \mathrm{~K}$, volatiles are by far the dominant solid species to condense (Barshay \& Lewis 1976). Among those, water dominates, first because oxygen is more abundant, and second because water condenses at higher temperatures than ammonia and methane. In a solar composition mixture, oxygen is more abundant than carbon by a factor 1.8 , to nitrogen by a factor 7.2 and to magnesium, silicium and iron by factors 12,15 and 15 , respectively (Asplund et al. 2009). We hereafter use water as a proxy for volatiles in general, an assumption that is minor compared to other sources of uncertainty.

From the phase diagram of $\mathrm{H}_{2} \mathrm{O}$ (Fig. 1) it is clear that with an atmospheric temperature above $1000 \mathrm{~K}$, the planet would be composed of supercritical water. If the planet follows an adiabat, it will remain in vapour form, transforming eventually into a plasma. If instead the planet had a surface temperature below the melting point of water (e.g. because it formed far from the central star), it would exhibit different high pressure forms of ice up to a regime where ice VII and ice X (for the massive icy planets) dominate.

The EOS used for water vapour is obtained from a combination of data obtained from a finite temperature molecular dynamics (FT-DFT-MD) simulation by French et al. (2009) and of the Sesame 7150 EOS (see Kerley 1972). The FT-DFT-MD data are used for $T=1000-10000 \mathrm{~K}$ and $\rho=2-7 \mathrm{~g} \mathrm{~cm}^{-1}$ as well as for $T=10000-40000 \mathrm{~K}$ and $\rho=5-15 \mathrm{~g} \mathrm{~cm}^{-1}$. Sesame 7150 data

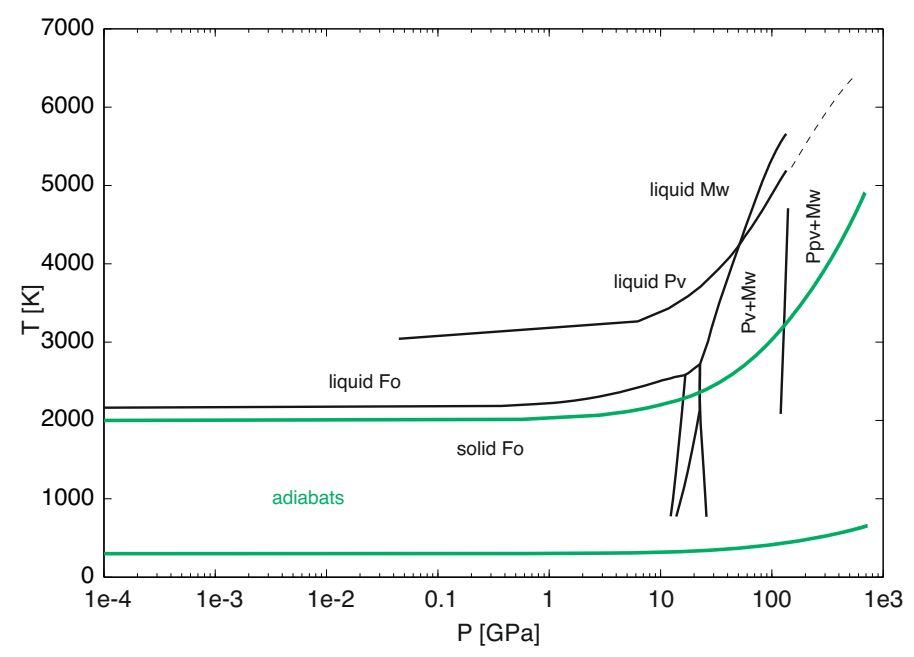

Fig. 2. Simplified $P-T$ phase diagram for relevant silicates on superEarths. The data are taken from Presnall (1995) for the Mg-silicate end member, with phase boundaries from solid forsterite (dominant in the upper mantle), to Earth's lower mantle materials, perovskite (pv) and magnesiowustite (mw). The melting curve for pv and mw, which remains controversial, is shown as well as an extrapolation of the pv's melting curve to higher pressures. The phase boundary of postperovksite (ppv) was calculated from Tsuchiya et al. (2004). Adiabats at $300 \mathrm{~K}$ and $2000 \mathrm{~K}$ and 1 bar are shown for reference.

are used elsewhere. The two EOS are joined by interpolation of isotherms.

\subsubsection{Silicates}

Although silicates are basically made of $(\mathrm{Mg}, \mathrm{Fe}) \mathrm{O}+\mathrm{SiO}_{2}$, the phase diagram relevant for the mantle is very complicated due to the different minerals that can be formed and the presence of iron and other minor elements $(\mathrm{Ca}, \mathrm{Al})$. We show the relevant phases for the magnesium end member in Fig. 2. The diagram shows the forsterite ( $\left.\mathrm{Fo}: \mathrm{Mg}_{2} \mathrm{SiO}_{4}\right)$, perovskite/post-perovskite (pv/ppv: $\mathrm{MgSiO}_{3}$ ), magnesiowustite (mw: $\mathrm{MgO}$ ) system. In addition, the upper mantle would also include the pyroxene phases $\left(\mathrm{Mg}_{2} \mathrm{Si}_{2} \mathrm{O}_{6}\right)$ ).

We show two adiabats calculated at $300 \mathrm{~K}$ and $2000 \mathrm{~K}$ and 1 bar for comparison. Both melting curves of pv and mw show a steep slope that can pose a barrier to the melting of the interior. Given that we do not know the melting behaviour of postperovskite or of $\mathrm{MgO}$ at high pressures, it remains unclear if the lower-most mantle of super-Earths can easily melt or not.

It should be noted that melting will depend on the amount of iron in the mantle (i.e. the magnesium number), but also on the abundance of minor species, something not included in Fig. 2. As an example, on Earth decompression melting can occur at temperatures around $1300 \mathrm{~K}$ (Hirschmann 2000). We do not attempt to determine the fraction of the planet's surface that may be molten, but note that it may be relatively large.

\subsubsection{Iron}

While forming a pure iron planet is very unlikely, evaporating the mantle of a Mercury-like planet might be possible. We show the phase diagram for iron in Fig. 3. Different phases of iron have been identified in the low pressure regime (Boehler 2000) with relative agreement. The $\epsilon$ phase seems to be the most relevant to Earth's core. A pure-iron planet might transition between 
D. Valencia et al.: Composition and fate of short-period super-Earths

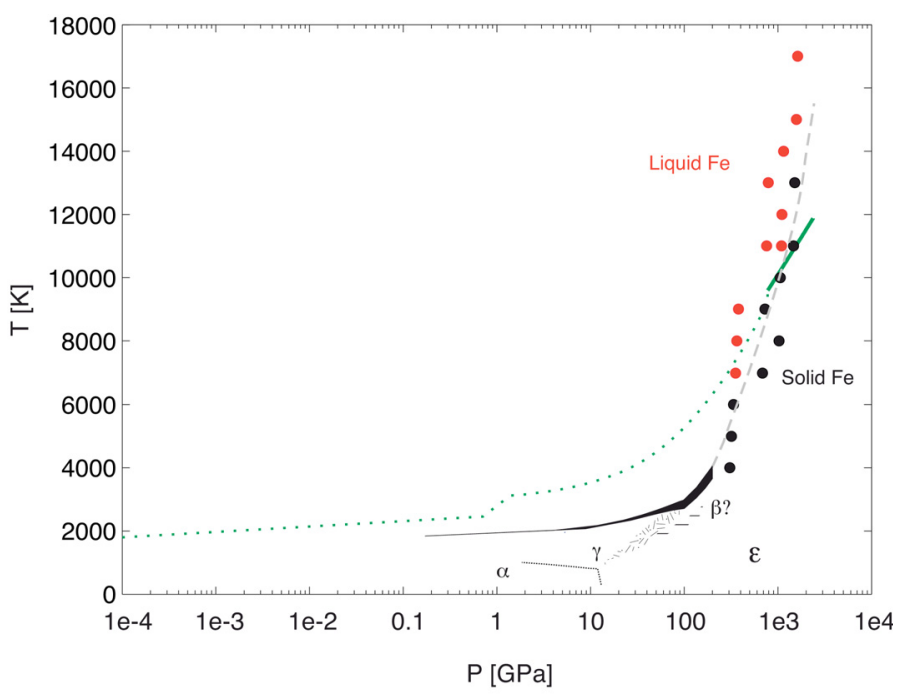

Fig. 3. $P-T$ phase diagram for iron. Values for pressures below $200 \mathrm{GPa}$ were adapted from Boehler (2000) and references therein. The black region shows the agreement in the melting curve of iron at relatively low pressures. Data points for melting in the high pressure regime of 306-1625 GPa are from Morard et al. (2009). Red points correspond to the liquid phase, while black points are solid Fe. The dashed curve is a melting line drawn to approximate the boundary suggested by the results from the ab-initio calculations. The temperature profile for an Earth-like CoRoT-7b is shown in green. The dotted part corresponds to the mantle, whereas the solid line corresponds to the core's temperature.

different phases of iron depending on the pressure-temperature profile. However, the high pressure regime in which most of the cores of super-Earths would be in, is still inaccessible to experiments. Thus it is unclear if there are any other higher pressure phases of iron unidentified at this point. One study (Morard et al. 2009) has reported the melting behaviour of iron in the tens of megabars pressure regime from ab-initio calculations (red and black symbols in Fig. 3). The melting boundary is quite steep, implying that pure-iron planets are likely to be mostly solid. However, planets with mantles have hotter interiors due to their insulating character.

\section{Inferring composition}

\subsection{CoRoT-7b as a rocky (iron+rock) planet}

\subsubsection{Description}

We first explore the case where the planet is of telluric composition. This implies a variety of compositions, from a pure magnesium-silicate planet (with no iron) to a pure iron planet. The former would yield the largest size for a rocky body, while the latter would be the smallest. Either case is unlikely. During the cooling of a protoplanetary disc, iron and silicates are condensed out at similar temperatures so that if iron is present, so are silicates, and visceversa, especially in large objects. Furthermore, the variety in structure for rocky planets includes those that are differentiated and undifferentiated. The former has a layered structure with the core composed mainly of iron, and in the case of Earth some nickel and a light alloy (McDonough \& Sun 1995) below a silicate mantle. The mantle can also incorporate iron within the oxide structure, replacing the magnesium site. Undifferentiated planets would have all of their iron content embedded in the mantle rocks. The amount of iron with respect to magnesium in the mantle (the magnesium

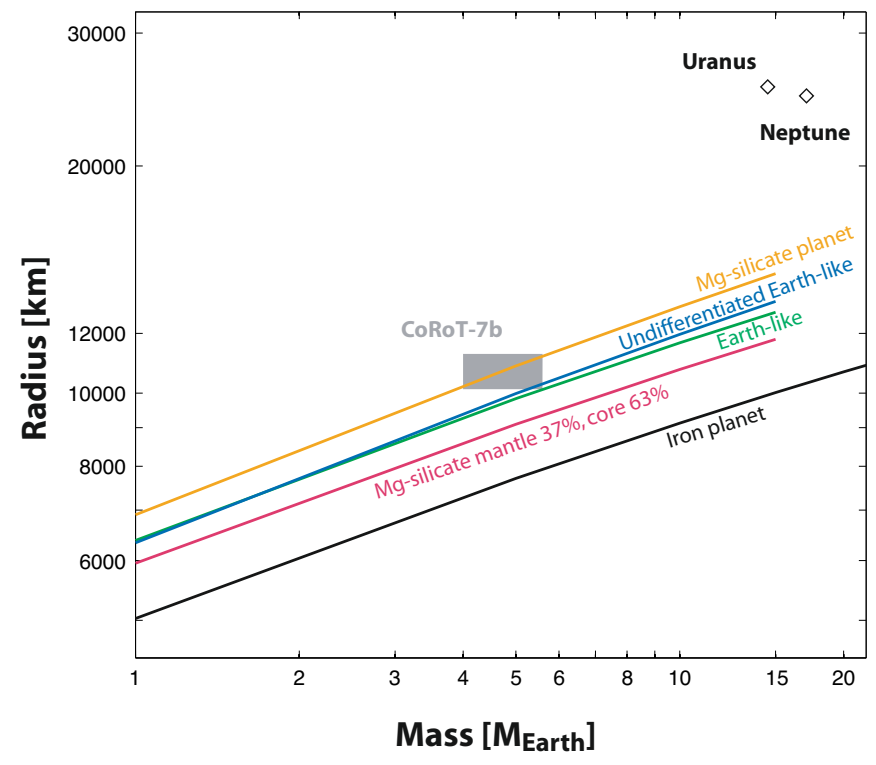

Fig. 4. Mass-radius relations for planets made of iron and rocks. The compositions considered are pure iron, $63 \%$ core and $37 \%$ mantle, Earth-like (33\% core and $67 \%$ mantle with $x_{\mathrm{Fe}}=0.1$ iron content), an undifferentiated planet with bulk Fe/Si $=2$ (Earth-like), and 100\% $\mathrm{Mg}$-silicate mantle planet. The surface temperature is taken to be $1800 \mathrm{~K}$. The shaded area corresponds to CoRoT-7b's measured radius and mass. Any combination of M-R that lies above the relation for pure $\mathrm{Mg}$-silicate planets (orange line) requires a composition that includes volatiles. Compositions with larger amounts of iron would progressively lie below this line.

number) speaks to the degree of differentiation of a planet and is a consequence of early formation, when the part of $\mathrm{Fe}$ that remained immiscible differentiated to form the core. The iron content $\mathrm{x}_{\mathrm{Fe}}=\mathrm{Fe} /(\mathrm{Mg}+\mathrm{Fe})$ for Earth has been estimated at 0.1 (McDonough \& Sun 1995), while for Mars it is calculated to be $0.20-0.25$ (Ohtani \& Kamaya 1992). For super-Earths this number may greatly vary, although due to a higher accretional energy, bigger planets may be expected to be differentiated.

On the other hand, the composition of planets can be compared by looking at bulk elemental ratios like Fe/Si. For Earth this number is considered to agree with that of CI chondrites (McDonough \& Sun 1995) and is 2. Although it is unclear if the planets should have the same Fe/Si bulk ratio as their host star, it is a reasonable assumption. Mercury is an anomaly in the solar system. However, its anomalously high iron content may be related to secondary formation processes like giant impacts and erosion, which may have dramatic effects on planetary compositions.

To infer CoRoT-7b's composition we considered different possibilities: 1) a pure Mg-silicate planet; 2) an Earth analog (i.e. a differentiated planet with $x_{\mathrm{Fe}}=0.1$, and a core that is $33 \%$ by mass); 3) an undifferentiated planet with the same bulk $\mathrm{Fe} / \mathrm{Si}$ ratio as Earth's, which we obtain with an iron content of $x_{\mathrm{Fe}}=0.76$ by mol; (4) a planet with no iron in the mantle and a core-mass fraction of $63 \%$ (i.e. a super-Mercury); and (5) a pure iron planet. The mass-radius relations are shown in Fig. 4.

To calculate the $\mathrm{Fe} / \mathrm{Si}$ ratio of the differentiated and undifferentiated planets we considered a mantle composed of $\frac{1}{2}\left(\mathrm{Mg}_{\left(1-x_{\mathrm{Fe}}\right)}, \mathrm{Fe}_{x_{\mathrm{Fe}}}\right)_{2} \mathrm{SiO}_{4}+\frac{1}{2}\left(\mathrm{Mg}_{\left(1-x_{\mathrm{Fe}}\right)}, \mathrm{Fe}_{x_{F e}}\right)_{2} \mathrm{Si}_{2} \mathrm{O}_{6}$ in the upper mantle and $\frac{3}{4}\left(\mathrm{Mg}_{\left(1-x_{\mathrm{Fe}}\right)}, \mathrm{Fe}_{x_{\mathrm{Fe}}}\right) \mathrm{SiO}_{3}+\frac{1}{4}\left(\mathrm{Mg}_{\left(1-x_{\mathrm{Fe}}\right)}, \mathrm{Fe}_{x_{\mathrm{Fe}}}\right) \mathrm{O}$, in the lower mantle and lowermost mantle (the post-perovskite region). In addition, we used a $\mathrm{Ni} / \mathrm{Fe}$ ratio of 17 and had a light alloy in 
A\&A 516, A20 (2010)

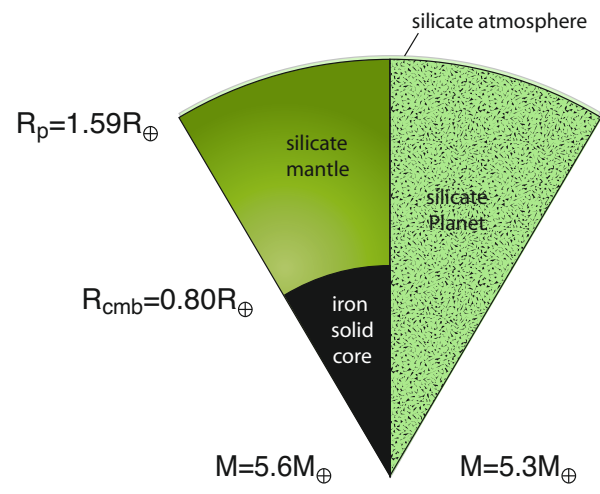

Fig. 5. Drawing to show the interior structure envisioned for a rocky planet. Two scenarios with $\mathrm{Fe} / \mathrm{Si}=2$ : (left) Earth-like ( $10 \%$ by mol of iron in the mantle and core-mass fraction of $33 \%$ ), or (right) undifferentiated.

the core of $8 \%$ by mass after Earth's composition (McDonough \& Sun 1995).

Because the largest radius for a rocky CoRoT-7b corresponds to the $\mathrm{Mg}$-silicate planet, any radius above this line reveals volatiles. Rocky planets with increasing amounts of iron content, whether differentiated or undifferentiated, will have a massradius relation lying progressively below the pure $\mathrm{Mg}$-silicate line. Coincidentally, this 'super-Moon' composition conforms to the smallest and largest mass of CoRoT-7b. However, this is unrealistic, as iron is expected to be present in some amount, so that the lower end of CoRoT-7b's mass range cannot be justified without the presence of volatiles. Massive planets mostly made of silicates and with very little iron ("super-Moons") are unlikely to exist: it is difficult to imagine how the special conditions that led to the formation of our Moon could also prevail for a planet 500 times more massive, and with the dissapearance of the massive iron-rich counterpart object (equivalent to the Earth).

The difference between differentiated and undifferentiated planets is that the latter are slightly larger and this effect becomes more noticeable with increasing mass. Our result agrees with that of Elkins-Tanton \& Seager (2008). The difference in radius for planets with $1,5,10$ and $15 M_{\oplus}$ is of $0.7 \%, 1.5 \%, 2.6 \%$ and $3.4 \%$ respectively. Thus, it seems implausible from mass and radius measurements to distinguish between a differentiated and undifferentiated planet. However, perhaps atmospheric evaporation of silicates indicating the amount of iron might help infer the state of differentiation.

We exemplify the different structures of an Earth-like composition and the equivalent undifferentiated planet in terms of the $\mathrm{Fe} / \mathrm{Si}$. For a fixed radius of $R=1.59 R_{\oplus}$ (a one sigma decrease in the measured radius) the differentiated and undifferentiated cases would have a mass of 5.6 and 5.3 respectively. Figure 5 depicts their different interiors.

Furthermore, the state of the core is shown in Fig. 3, where we show the temperature profile for an Earth-like composition including concentration of radioactive sources and an age of $1.8 \mathrm{~Gy}$. The core's temperature lies right at the melting boundary, so that given the uncertainties in temperature structure, there is a possibility of an outer liquid core. These uncertainties include the exact concentration of radioactive sources (perhaps more potassium), the existence of a boundary layer right above the core, and even possibility of a layered mantle due to the different viscosities between perovskite and postperovskite. Given the very steep behaviour of the iron solidus, it seems unlikely that massive terrestrial planets could have liquid cores, especially those with a surface temperature that allows for liquid water.

\subsubsection{The atmosphere}

Because of the high irradiation the planet is subject to, its surface may be heated to extremely high temperatures. Given the star's characterisics $\left(T_{\mathrm{eff}}=5275 \mathrm{~K}, R_{\star}=0.87 R_{\odot}\right)$ and planet's semi-major axis ( $\left.a=4.27 R_{\star}\right)$, the effective equilibrium temperature at the planet's substellar point is (assuming a zero albedo) $T_{\mathrm{eq}}^{\text {sub }}=2570 \mathrm{~K}$. Distributed evenly on the planet's surface, this temperature is $T_{\mathrm{eq}}^{\text {global }}=1820 \mathrm{~K}$. Even though the exact temperature depends on the emissivity and albedo, it is clear that silicates (or iron if present at the surface) should be molten by the intense heat, at least on the portions of the planet with temperatures above $2000 \mathrm{~K}$ (see Figs. 2 and 3).

A first consequence of a molten surface is that volatiles should be efficiently outgassed from the planetary interior (e.g. Schaefer \& Fegley 2007). However, because the total mass of those volatiles would be as small as at most a few $10 \%$ of the planet's mass, this is a temporary effect and given the significant mass loss, the massive atmosphere thus formed should disappear quickly (on a timescale of $10^{6}-10^{8} \mathrm{yrs}$, based on the arguments in Sect. 2). The remaining planet should then contain only refractory material, with vapour in equilibrium with the lava, and an evolving composition as a function of the mass loss.

Specific models for the chemistry of the atmosphere of evaporating silicate super-Earths (Schaefer \& Fegley 2009) indicate that a planet like CoRoT-7b with a composition similar to that of the bulk silicate Earth would have an equilibrium atmosphere with a pressure initially between $10^{-6}$ and $10^{-2}$ bars (corresponding to our range of extreme temperatures). The evaporating vapour atmosphere should be mainly composed of $\mathrm{Na}$, then $\mathrm{SiO}, \mathrm{O}$ and $\mathrm{O}_{2}$, then $\mathrm{Mg}$, as the less refractory species are progressively lost. Schaefer \& Fegley (2009) find that the pressures decrease by about one order of magnitude when $\mathrm{Na}$ is lost, and then by a further two to three orders of magnitude at $90 \%$ of total erosion.

With such a thin atmosphere, the planetary radius measured from the transits can be considered as that of the solid/liquid surface of the planet.

\subsubsection{Limits to the mass loss?}

Compared to the arguments presented in Sect. 2, the planet's erosion may be reduced if (i) UV photons are not fully absorbed in the atmosphere, but hit the surface of the planet, or (ii) the "supply" of atmosphere is slowed by the need to deliver heat to pass the latent heat barrier.

The photoionization cross-section of atomic species like $\mathrm{H}$, $\mathrm{O}, \mathrm{Fe}, \mathrm{Mg}, \mathrm{Si}$ is between $\sigma_{\mathrm{UV}} \approx 10^{-19}$ and $10^{-17} \mathrm{~cm}^{2}$ in the 10-50 eV energy range (Verner et al. 1996). The unit optical depth for UV photons corresponds to a pressure $P_{\mathrm{UV}}=$ $\mu m_{\mathrm{p}} g / \sigma_{\mathrm{UV}}$, where $\mu$ is the mean molecular weight of the atmosphere, $m_{\mathrm{p}}$ the proton's mass and $g$ the planet's gravity. Using $g \approx 1000 \mathrm{~cm} \mathrm{~s}^{-2}$ and $\mu \approx 10 \mathrm{~g}$ one finds $P_{\mathrm{UV}} \approx 10^{-7}$ to $10^{-9}$ bar. (For comparison, $P_{\mathrm{UV}} \approx 10^{-9}$ bar for gas giants - see MurrayClay et al. 2009.)

Because those values of $P_{\mathrm{UV}}$ are much smaller than those of the vapour pressure estimated above, it appears that the equilibrium vapour atmosphere is able to efficiently absorb stellar UV photons that will drive the escape from the planet. Note however 
that the precise rate of escape depends on cooling processes and hydrodynamical modelling much beyond the scope of this work.

Let us now consider whether a bottleneck to the escape may be caused by the need to vaporize material that is initially solid or liquid. In order to do so, we balance the energy required for the sublimation at a rate $\dot{M}_{\text {sub }}$ with the absorbed heat flux $\pi R_{\mathrm{p}}^{2} F_{\star}$, where $F_{\star}$ is the stellar irradiation. Note that $F_{\star}$ is formally the stellar flux that reaches the ground, but given the thinness of the atmosphere, this is equivalent to the irradiation flux at the top of the atmosphere. The sublimation rate can hence be written as

$\dot{M}_{\text {sub }}=\frac{\pi R_{\mathrm{p}}^{2} F_{\star}}{\mathcal{L}_{\text {sub }}}$,

where $\mathcal{L}_{\text {sub }}$ is the latent heat of sublimation. Using $\mathcal{L}_{\text {sub }} \approx$ $10^{9}-10^{10} \mathrm{erg} \mathrm{g}^{-1} \mathrm{~K}^{-1}$ (typical for iron and silicates), we find $\dot{M}_{\text {sub }} \approx 10^{17}$ to $10^{18} \mathrm{~g} \mathrm{~s}^{-1}$, i.e. at least six orders of magnitude higher than the mass loss we previously derived. Hence, there is no mass loss suppression due to latent heat effects.

We thus conclude that at this orbital distance, less than five stellar radii away from its star, the planet should be eroding even if made of the most refractory materials!

\subsubsection{Evaporation of a rocky CoRoT-7b}

We now investigate the possible precursors of CoRoT-7b, given that its mantle is subjected to considerable erosion. Calculations of this erosion depend on the orbital evolution of the planet, the decrease in stellar EUV flux, but mostly on the change in bulk density as layers (or perhaps selective components) of the planet are stripped away. In this section we are mostly concerned with the effect of changing density. We integrated Eq. (1) backward in time to assess the mass loss experienced and calculated the composition of the precursors. We used the internal model to obtain the planet's structure at each time step and calculated its density. The chosen efficiency for this calculation is $\epsilon=0.4$. We considered two present compositions: a "super-Moon" and an Earthlike composition. They correspond to the lightest and densest compositions for a rocky CoRoT-7b. The results are shown in Fig. 6 and exemplify the role of changing density.

We found that small planets suffer a greater atmospheric loss due to the inverse dependence on average density. Also, as planets lose their silicate mantles, their average density either increases (for small planets) or stays relatively constant (for massive planets) owing to a cancelling effect between a reduction in size and mass. Thus, the rate of atmospheric loss decreases through time. Assuming $\epsilon=0.4$, we calculated the amount of mass lost to be 3-4 $M_{\oplus}$, so that if formed rocky, CoRoT-7b was initially a $\sim 8 M_{\oplus}$-planet with a core that was at most $22 \%$ by mass and $\mathrm{Fe} / \mathrm{Si}=1.28$. This is probably an upper limit, given that studies of the hydrodynamic loss of a silicate-rich atmosphere have never been done and may lead to a lower $\epsilon$ values. Plus, we do not consider probable inward migration, or uncertainties in the EUV flux.

\subsection{CoRoT-7b as a vapour planet}

\subsubsection{Description and possible origin}

Given CoRoT-7b's relatively large mass, it should have originally accreted a significant mass of gases, i.e. hydrogen and helium (e.g. Ikoma \& Genda (2006)), and volatiles (a.k.a "ices") (e.g. McNeil \& Nelson (2010)). Given the present extreme stellar irradiation, one would expect volatiles, if present on the planet,

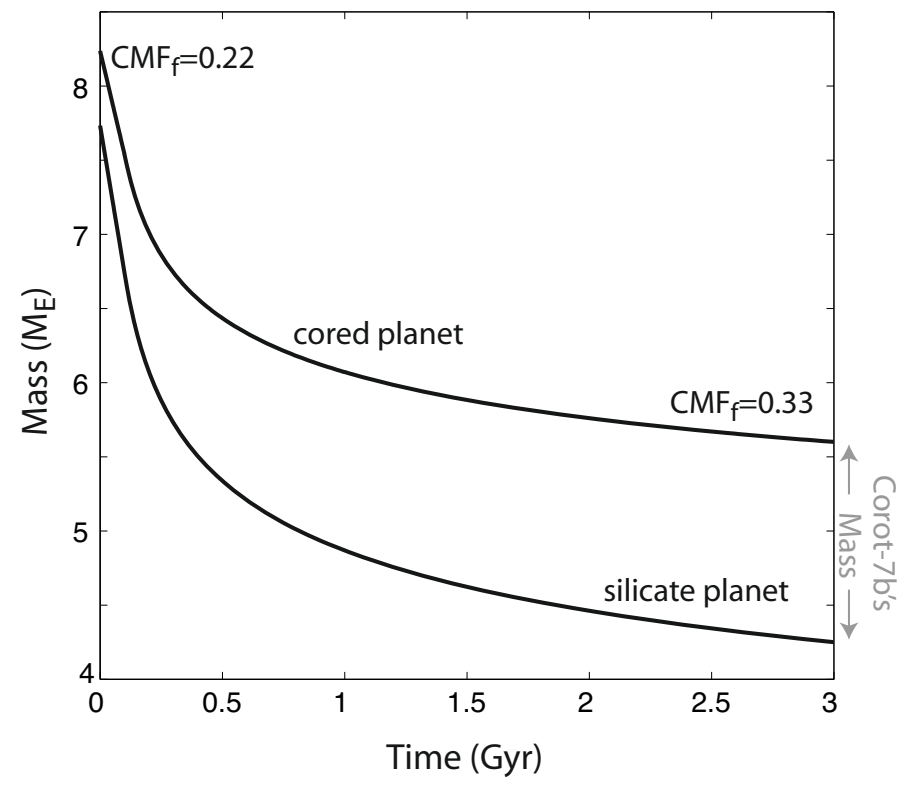

Fig. 6. Evolution in mass for CoRoT-7b for a rocky composition. Two present compositions were considered: a "super-Moon" (little or no iron content), and an Earth-like composition (33\% iron core, 67\% silicate mantle), corresponding to the lightest and densest cases for a rocky CoRoT-7b, respectively. The initial and final proportions of core mass fraction are shown. The mass loss is more significant early on due to its inverse relation to density. The mass loss is calculated from Eq. (3), with $\epsilon=0.4$.

to be in vapour form (see Sect. 3.2). However, we first examine the alternative hypothesis that if the planet migrated from large orbital distances (e.g. Lin et al. 1996), water had time to cool and solidify.

Let us consider a planet of $M_{\mathrm{p}}=10 M_{\oplus}$ made mostly of water (for simplicity). The gravitational energy transformed into internal energy during its formation is $E_{\mathrm{i}} \approx 3 / 10 G M_{\mathrm{p}}^{2} / R_{\mathrm{p}}$. In the absence of irradiation (if far enough from the star), the planet is initially made of vapour. If vapour is present in the photosphere (i.e. without an optically thick layer of hydrogen and helium), it will maintain a high atmospheric temperature (and therefore a rapid cooling) until complete condensation of water onto the interior is reached. Given an effective temperature (as obtained from the temperature at which the saturated vapour pressure (e.g. Emmanuel 1994) is equal to the photospheric pressure, $\left.P_{\text {vap }}(T)=2 / 3 \mathrm{~g} / \kappa\right)$, an upper limit to the time to solidification is $\tau_{\text {solid }}=E_{\mathrm{i}} /\left(4 \pi R_{\mathrm{p}}^{2} \sigma T_{\mathrm{eff}}^{4}\right)$, i.e. for $R_{\mathrm{p}} \approx 15000 \mathrm{~km}, \tau_{\text {solid }} \approx 100 \mathrm{Ma}$ when using a low opacity $\kappa=10^{-2} \mathrm{~cm}^{2} \mathrm{~g}^{-1}\left(T_{\text {eff }} \approx 320 \mathrm{~K}\right)$. On the other hand, if ice grains and/or water droplet, in the atmosphere prevent it from cooling efficiently, $\kappa \approx 10^{2} \mathrm{~cm}^{2} \mathrm{~g}^{-1}$ ( $T_{\text {eff }} \approx 210 \mathrm{~K}$ ) so that $\tau_{\text {solid }} \approx 1 \mathrm{Ga}$.

At least in theory there is thus a possibility that CoRoT-7b is a planet that was formed at large distances from the star, had sufficient time (hundreds of millions of years) to solidify before being brought to an orbital distance of $0.017 \mathrm{AU}$, where ice would be sublimating again.

However, we believe that this is very unlikely for two reasons: First, a planet that massive should accrete some hydrogen and helium (Ikoma \& Genda 2006), and even only a few bars of these species would mean that water would still be in vapour form in the interior at smaller effective temperatures than estimated above in the pure water case (a situation similar to that of Uranus and Neptune). This would imply a (much) slower cooling, and thus retaining water in vapour form for a longer time. 


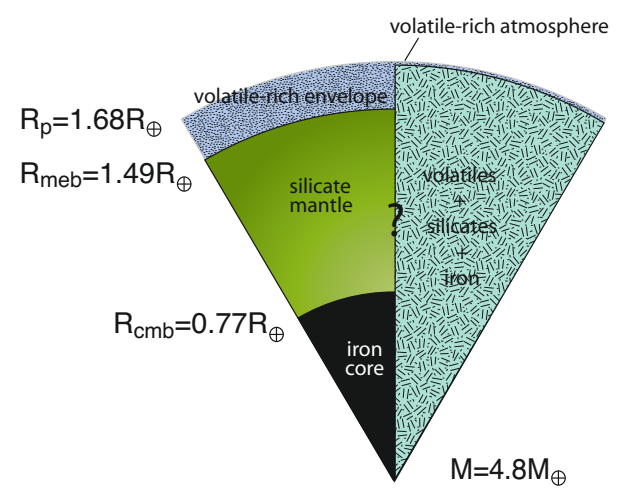

Fig. 7. Drawing to show the interior structure envisioned for a planet made of iron, rocks and volatiles in vapour form (possibly with $\mathrm{H}-\mathrm{He}$ ).

Note that invoking a putative evaporation of an outer hydrogen atmosphere would require an increased irradiation, which would also be unfavourable to the rapid cooling of the planet. Second, although possibilities of slow/delayed migration exist (e.g. Wu \& Murray 2003), most of the migration scenarios require "help" from the protoplanetary disc, and therefore a migration in the first millions of years (e.g. Moorhead \& Adams 2005). Below we therefore only consider the possibility that ices are in vapour form. Figure 7 depicts the two possibilities that we envision: a fully differentiated planet with iron, silicates and an extended envelope of vapour, or a fully homogeneous planet in which iron, silicates and volatiles (in vapour form) are thoroughly mixed.

\subsubsection{Constraints on the presence of vapour}

To estimate possible amounts of vapour compatible with the measurements of CoRoT-7b, we proceed as follows: first, for simplicity, we only considered the case of a solid/liquid interior that is "Earth-like" in composition (33\% iron core, $67 \%$ mantle rock) and surrounded by an envelope of volatiles in vapour form. Our calculations of interior models for the iron+rock part as a function of its mass $M_{\mathrm{Fe}+\mathrm{R}}$ and outside pressure $P_{0}$ are found to yield radii of the order of

$$
\begin{aligned}
R_{\mathrm{Fe}+\mathrm{R}}= & 9800 \mathrm{~km}\left(\frac{M_{\mathrm{Fe}+\mathrm{R}}}{5 M_{\oplus}}\right)^{0.28+0.02 \sqrt{M / 5 M_{\oplus}}} \\
& \times 10^{-\left[\log _{10}\left(1+P_{0} / \sqrt{M / 5 M_{\oplus}} / 7\right]^{3}\right.}
\end{aligned}
$$

where $P_{0}$ is in GPa units. The relation is an approximation found to be accurate to $\pm 0.5 \%$ for $M_{\mathrm{Fe}+\mathrm{R}}$ between 1 and $15 M_{\oplus}$ and $P_{0}$ up to $10^{3} \mathrm{GPa}(=100 \mathrm{Mbar})$. The approximation is accurate to $\pm 3 \%$ to $10^{4} \mathrm{GPa}$.

The size of the planet with vapour is found by calculating the evolution of an initially adiabatic planet with a specific entropy equal to that of vapour at $10 \mathrm{bar}$ and $2500 \mathrm{~K}$. This initial state is chosen as representative of any "hot start", since any evolution from still higher entropies would have been fast. We neglected any possible orbital evolution of the planet.

The evolution is characterized by the rapid growth of a radiative zone just below the atmospheric boundary, similarly to what is obtained for giant exoplanets (Guillot 2005). This zone quickly becomes isothermal and extends down to pressures around $10 \mathrm{kbar}$ and temperatures $\sim 3000 \mathrm{~K}$. At those pressures and temperatures, the rapid rise in radiative opacities implies that any further extension of the radiative region must wait for a large reduction of the intrinsic luminosity, implying a slow cooling.

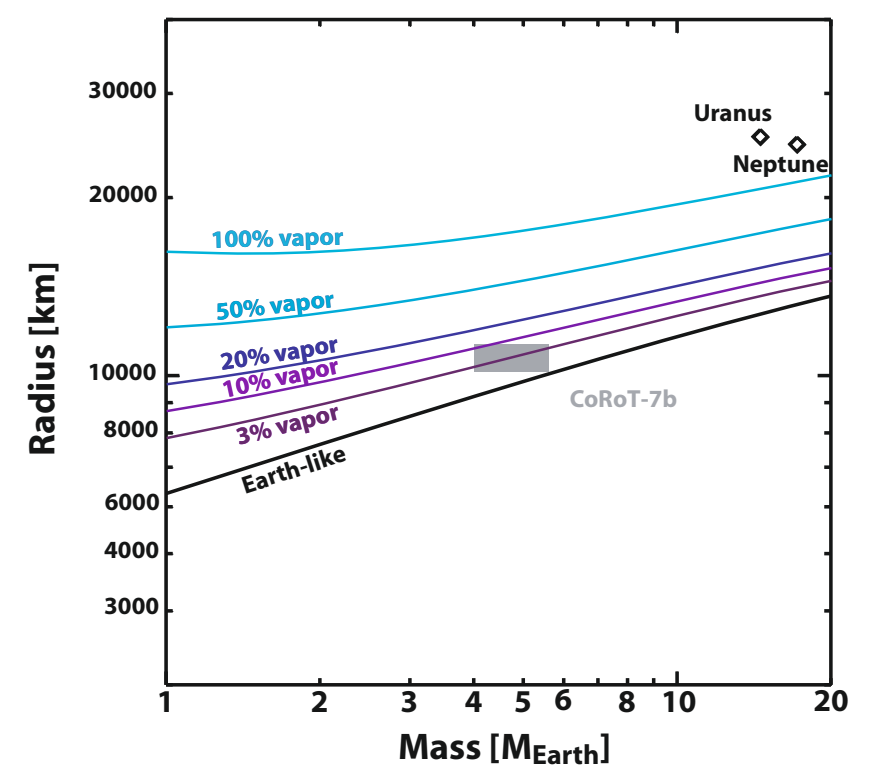

Fig. 8. Radius as a function of mass for a planet made of iron, rocks and vapour. The "Earth-like" line corresponds to the limiting value of a solid Earth-like planet with a mass fraction of iron to rocks of 33\% and without vapour. Other lines corresponds to radii for planets with an Earth-like interior and a vapour $\left(\mathrm{H}_{2} \mathrm{O}\right)$ envelope, with a ratio of the mass of the vapour envelope to the total planetary mass between $3 \%$ and $100 \%$, as labelled. The models with vapour have been evolved for $1 \mathrm{Ga}$ using our fiducial opacity table (see text). Radii correspond to the 10 bar level.

This implies that results should be relatively robust with regard to the uncertainties in the initial state, opacities, age...etc.

Figure 8 shows the resulting planetary radii after $2 \mathrm{Ga}$ of evolution for various mass fractions of vapour in the planet. The presence of an atmosphere of vapour is found to affect the structure and size of the planet significantly. We find that the upper limit on the amount of vapour present in CoRoT-7b is $\sim 10 \%$, i.e. the equivalent to about $0.5 M_{\oplus}$. Because the cooling and contraction occurs rapidly, we found that this value is robust and does not change by more than a few percent when considering cooling times between 0.1 and $10 \mathrm{Ga}$. Given the evaporation rate calculated in Sect. 2, this implies that such an atmosphere would last for another $\mathrm{Ga}$ or so. Hence, this is a reasonable possibility. Our best vapour-planet model for CoRoT-7b with a total mass of $4.8 M_{\oplus}$, has a vapour envelope that is $3 \%$ of the total mass and $12 \%$ of the total radius (see Fig. 7). For this model, the envelope is close to being isothermal: the transition between the vapour envelope and the silicate mantle is at a temperature of $2900 \mathrm{~K}$ for a pressure of $14 \mathrm{GPa}$.

\subsubsection{Constraints on the presence of hydrogen and helium}

With the same method we derived constraints on the amounts of hydrogen and helium that may be present. Figure 9 shows that the presence of an envelope hydrogen and helium leads to a very significant increase in the size of the planet. Because of the low gravity and high compressibility of the envelope, we found that planets with smaller masses have larger radii if they contain a $\mathrm{H}-\mathrm{He}$ envelope and are significantly irradiated, except when the envelope to core mass ratio is so small that the envelope is still tightly bound by gravity to the Earth-like nucleus. We derived that any hydrogen-helium envelope in CoRoT-7b must be less than $0.01 \%$ of the total planetary mass. Note that for such small 


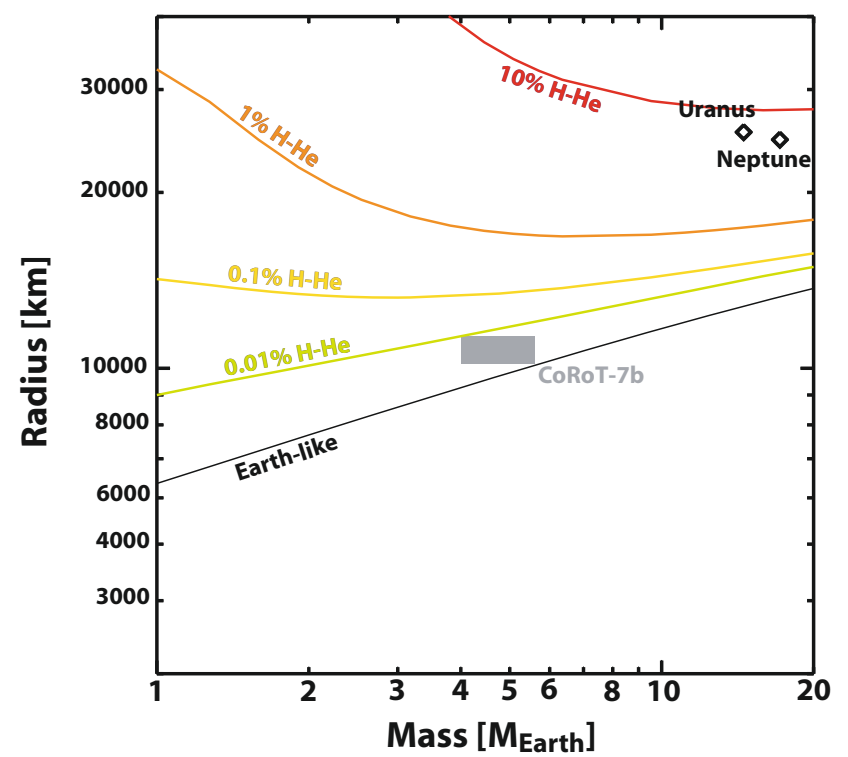

Fig. 9. Radius as a function of mass for a planet made of iron, rocks, and hydrogen and helium in solar proportions. The "Earth-like" line corresponds to the limiting value of a planet without vapour. Other lines correspond to planets with hydrogen and helium envelopes having total mass fractions between $0.01 \%$ and $100 \%$ (planet made only of hydrogen and helium), as labelled. (See Fig. 8.)

envelopes the structure is isothermal (the envelope to silicate transition occurs at a temperature that is within a few Kelvins of the assumed 10 bar temperature). If CoRoT-7b would now possess such an envelope, it would evaporate in only $1 \mathrm{Ma}$. We therefore estimate that CoRoT-7b cannot possess a hydrogenhelium atmosphere.

\subsubsection{Possibility of an undifferentiated structure}

We have thus far assumed a differentiated structure (i.e., iron/rocks/volatiles or hydrogen-helium). While this is indeed verified for relatively small and cool planets (from Ganymede to the Earth), the question arises for planets that may be large and hot enough for their interior to be predominantly molten. Molten silicate and water are known to be miscible with each other at pressures above a few GPa and temperatures $>\sim 1000 \mathrm{~K}$ (Shen \& Keppler 1997; Mibe et al. 2007). Above $10000 \mathrm{~K}$, silicate and iron are also no longer immiscible (Stevenson 2008). Hydrogen, helium and water will mix as well at high enough temperatures. Indeed, Uranus and Neptune appear to be only partially differentiated, with an outer hydrogen-helium gaseous envelope that contains a high abundance of at least one of the volatile components, i.e. methane, an inner dense envelope, which appears to be mostly made of high-pressure and high-temperature "ices", probably mixed with rocks, and a central dense nucleus (probably made of rocks and/or iron) with a mass of the order of $1 M_{\oplus}$ or smaller (Hubbard et al. 1995). Some interior models compatible with the observed $J_{2}$ and $J_{4}$ values even include an inner envelope composed of a mixture of hydrogen-helium and "ices" (Marley et al. 1995).

Specific studies are required to solve this problem. We stress that our limits on the presence of a gaseous or vapour envelope cannot be applied as constraints on the presence of these gases and/or volatiles in the mantle. If the planet is undifferentiated, a larger component of gases and volatiles may be present than derived in the previous sections. (This is because the higher mean molecular weight will at some point prevent the planet from inflating thermally as much as if all light species were present in an outer envelope.) For robust conclusions concerning undifferentiated super-Earths in general, detailed models beyond the scope of this paper are needed. The lack of adequate equations of state and relevant opacities are difficult limitations to overcome.

In any case, we note that the mean temperature in the atmosphere should be low enough to maintain a low abundance of silicate species there. Specifically, if we assume a photospheric level close to the mean equilibrium temperature, $T_{0}=$ $1800 \mathrm{~K}$, the saturation pressure of $\mathrm{MgSiO}_{3}$, representative of rock species, for that temperature is $P_{\mathrm{s}} \approx \exp (-58663 / T+$ $25.37) \approx 7 \times 10^{-4}$ bar (Lunine et al. 1989), i.e. much lower than the photospheric pressure $P_{0}=(2 / 3)(g / \kappa) \approx 1$ bar for $\kappa=10^{-2} \mathrm{~cm}^{2} \mathrm{~g}^{-1}$. We thus envision that the outer atmosphere is rich in volatiles, leading to a preferential escape of these even in the homogeneous interior case (see Fig. 7).

\subsubsection{CoRoT-7b as an evaporated ice or gas giant?}

We now examine whether CoRoT-7b may have been formed by outstripping a gas giant or an ice giant from its envelope, leaving a planet with little or no gaseous envelope. In order to do so, we first calculated an ensemble of evolution models with a constant total mass and a constant composition. This ensemble of models is characterized by a central seed of Earth-like composition of $5 M_{\oplus}$ and variable total masses (from 10 to about $\left.120 M_{\oplus}\right)$. The combined mass and thermal evolution of a planet with mass loss was then calculated by noting that for each planetary mass and central specific entropy a given planetary radius corresponds, and therefore a given mass loss, and that central entropy should be conserved during mass loss

$$
\begin{aligned}
\frac{\mathrm{d} M}{\mathrm{~d} t} & =f\left(\rho_{\mathrm{p}}\right), \\
\frac{\mathrm{d} S_{\mathrm{c}}}{\mathrm{d} t} & =\left(\frac{\partial S_{\mathrm{c}}}{\partial t}\right)_{M},
\end{aligned}
$$

where $f\left(\rho_{\mathrm{p}}\right)$ is a function of the planet's density provided by Eq. (3), and the loss of central entropy $S_{\mathrm{c}}$ is calculated from individual evolution models with fixed mass.

Because evaporation is highly dependent on the planetary density, the choice of initial conditions affects the results directly. Here, we only highlight reasonable possibilities for the origin and fate of CoRoT-7b. We assumed that our planets filled their Roche lobe when formed, and allowed them to contract for $10 \mathrm{Ma}$ before turning on mass loss, using Eq. (1). This reproduces that the planet should be protected from mass loss during the protoplanetary disc phase, but should have begun its contraction and loss of entropy. The result also depends on the planet's orbital history, as a planet that is initially far from its star tends to contract faster. We therefore examine two cases: a) one where the planet is assumed to form in situ and remain at its present orbital distance $(0.017 \mathrm{AU})$ throughout its existence; b) one where its first $10 \mathrm{Ma}$ of existence are spend at larger orbital distances, i.e. $\sim 0.08 \mathrm{AU}$ (corresponding to an equilibrium 10 bar temperature of $1000 \mathrm{~K}$ ), before it is suddenly brought to its present orbital distance (and an equilibrium 10 bar temperature of $2500 \mathrm{~K}$ ).

The results are shown in Fig. 10. Vapour planets tend to be relatively compact and suffer significant, but limited mass loss. For example, present observations would be compatible with a vapour planet that was initially about $12 M_{\oplus}$ and that lost $97 \%$ of its vapour envelope in the "in situ" case. For this type of 

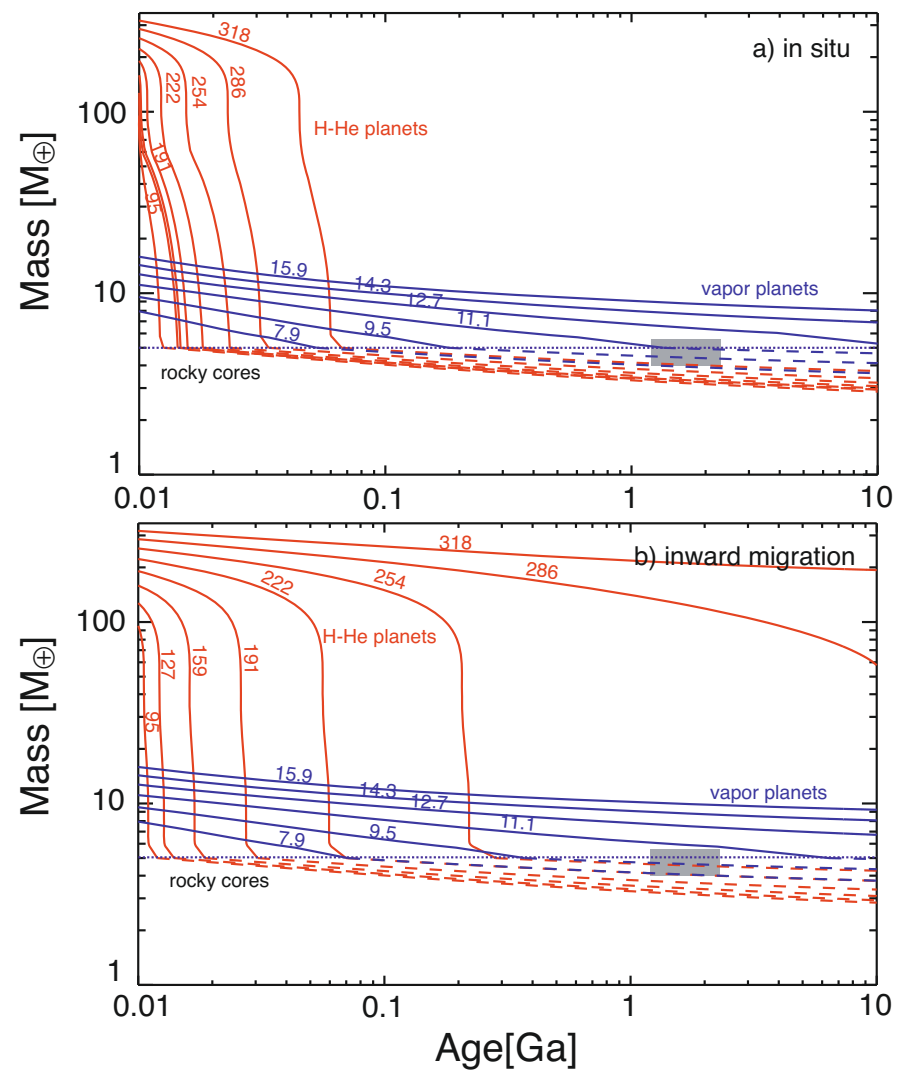

Fig. 10. Evolution of the mass of hypothetical CoRoT-7b precursors as a function of time. Two types of precursors are shown: planets with an extended hydrogen-helium envelope (red), and planets with a vapour (i.e. water) envelope. Labels indicate initial masses in Earth masses. All cases assume an inner $5 M_{\oplus}$ Earth-like core. Plain lines indicate planets that still possess an extended envelope. Dashed lines correspond to (evaporating) bare rocky cores. The top panel assumes no migration of the planet (maximal mass-loss). The bottom panel assumes a contraction of an initially extended planet during $10 \mathrm{Ma}$ at $\sim 0.08$ AU before migrating to its present location. The present mass and age of CoRoT-7b is indicated as a grey rectangle. A maximal, energy-limited evaporation is assumed (see text).

planet, the situation remains very similar when considering the "inward migration" scenario: the additional cooling only makes the vapour envelope slightly more compact so that the "ideal" precursor mass decreases to about $10 M_{\oplus}$. The situation is different for hydrogen-helium planets, because as was shown in Fig. 9 they tend to be very tenuous and loosely bound to the rocky nucleus. For example, we found that for planets at 0.017 AU filling their Roche lobe, the evaporation proceeds faster than the contraction and always lead to the complete loss of the envelope, even for initial masses as high as the mass of Jupiter. The top panel of Fig. 10 shows that even after $10 \mathrm{Ma}$ of evolution without mass loss, a Jupiter-mass planet is stripped of its entire envelope in less than $80 \mathrm{Ma}$. If the planet is allowed to contract at larger orbital distances (our "inward migration" case), the resulting planet is more compact, and possible precursors to CoRoT-7b are planets less massive than about $270 M_{\oplus}$. Thus, this shows that the evolution of layered planets may be very different and lead to a much more significant mass loss than when considering homogeneous planets for which contraction is approximately modelled by using simple power laws (Lammer et al. 2009). Of course, the numbers that we derived may vary quite significantly depending on the chosen formation scenario and dynamical evolution of the planet, but at least they show that CoRoT-7b could have lost tens of Earth masses in hydrogen and helium and that it is not impossible that it was initially a gas giant.

Our models are based on the assumption of the presence of distinct layers. An undifferentiated planet would evaporate at a quantitatively different rate, but we believe that qualitatively the situation would be very similar: it would preferentially lose its light elements (gases and volatiles) and at a rate that would be directly related to the proportions of hydrogen and helium and volatiles that the planet contains.

From the point of view of interior and evolution models, there is thus a range of possibilities to explain the characteristics of CoRoT-7b: it may have been initially a gas giant planet that was eroded to its dense rocky interior, it may have been a Uranus-like "ice giant", which would have lost most or all of its volatiles, or may have been always a rocky planet with no ice or hydrogen and helium.

\section{Conclusion}

CoRoT-7b is the first of possibly many extreme close-in superEarths that will be discovered in the near future. We have shown that the atmospheric escape for this type of planets is expected to be high, within an order of magnitude of that of HD 209458b, and mostly independent of composition. A simple analysis showed that for CoRoT-7b the mass already lost to escape would be of the order of $\sim 4 M_{\oplus}$ for silicate-iron planets, or $\sim 10-100 M_{\oplus}$ if the planet initially contained a massive water (hydrogen-helium) envelope.

Given the observational constraints on its size and mass, CoRoT-7b is best fitted by a rocky planet that would be significantly depleted in iron relative to the Earth. Such a massive "super-Moon" is very unlikely to form. However, a one sigma increase in mass $\left(5.6 M_{\oplus}\right)$ and one sigma decrease in size $\left(1.59 R_{\oplus}\right)$ would make the planet compatible with an Earth-like composition (33\% iron, $67 \%$ silicates). Such a rocky planet would have a thin vapour silicate atmosphere. We estimated that this atmosphere should be thick enough to efficiently capture stellar UV photons, therefore yielding a significant mass loss, possibly close to the rate obtained from energy-limiting considerations. Another possibility is that the planet was initially an ice giant that lost most of its volatile content. We calculated the maximum volatile envelope (in the form mostly of water vapour) for CoRoT-7b to be $10 \%$ by mass, with a best fit solution at $3 \%$. We also constrained a possible hydrogen-helium outer envelope to be less than $0.01 \%$ by mass. In both cases, these numbers were derived assuming the presence of an inner rock/iron core of Earth-like composition. The precursor of such a planet may be a Uranus-like ice giant, or it may have been a more massive gaseous planet: this is because gaseous planets at very short orbital distances may be extremely extended and have their envelope only weakly bound to the central rock core. Given the fast planetary erosion, we estimated that the vapour envelope that we derived to fit the present models would last up to $1 \mathrm{Ga}$ before its complete erosion. For a hydrogen-helium envelope, this survival time is only $1 \mathrm{Ma}$. This therefore implies that CoRoT-7b cannot contain a hydrogen-helium envelope. It is the first time that such a conclusion can be drawn for an exoplanet. A caveat is, however, that these estimates are based on the hypothesis that the structure of the planet is in the form of distinct layers with the light species on top. This may not be the case, in particular because water and silicates appear to mix efficiently at modest temperatures and pressures (above $1000 \mathrm{~K}$ and a few GPa), in which 
case potentially more volatiles may be "hidden" in the planetary interior. This is probably not a concern for hydrogen and helium, because they should be mostly on top of the rocky/icy nucleus already at the time of the planet formation. We showed with specific models that the undifferentiation of iron with respect to silicate does not significantly affect the planetary structure either. When it comes to water and silicates, the effect may be more pronounced because of their different densities and thermal properties. Specific studies including proper equations of state and opacities are required to progress in this respect.

Parallel to this, the mass loss that we inferred implies that there is a possibility to probe for the composition of the outer shell of the planet by measuring the composition of the extended planetary exosphere. The star CoRoT-7 $(V=11.7)$ is significantly fainter than HD $209458(V=7.65)$, so that the measurement is challenging. However, as a detection of escaping $\mathrm{H}, \mathrm{C}$ and $\mathrm{O}$ was possible for the planet HD 209458b (Vidal-Madjar et al. 2003, 2004) this yields great hopes for similar measurements for CoRoT-7b and close-in super-Earths in the near future.

Acknowledgements. This research was carried out as part of a Henri Poincare Fellowship at the Observatoire de la Côte d'Azur to DV. The Henri Poincare Fellowship is funded by the CNRS-INSU, the Conseil General des AlpesMaritimes and the Rotary International - District 1730. M.I. got financial support from the Program for Promoting Internationalization of University Education from the Ministry of Education, Culture, Sports, Science and Technology, Japan, and from the Plan Pluri-Formation OPERA. The authors acknowledge CNES and the CNRS program Origine des Planètes et de la Vie for support. The authors further thank the CoRoT community, Guillaume Morard, Didier Saumon, Bruce Fegley and Helmut Lammer for discussions and sharing results in advance of publication, and the anonymous referee for helping to improve this manuscript.

\section{References}

Adams, E., Seager, S., \& Elkins-Tanton, L. 2008, ApJ, 673, 1160

Alexander, D. R., \& Ferguson, J. W. 1994, ApJ, 437, 879

Asplund, M. N., Grevesse, A. J., \& Sauval, P. S. 2009, ARA\&A, 47, 481

Barshay, S. S., \& Lewis, J. S. 1976, ARA\&A, 14, 81

Boehler, R. 2000, Rev. Geophys., 38, 221

Elkins-Tanton, L. T., \& Seager, S. 2008, ApJ, 688, 628

Emmanuel, K. 1994, in Atmospheric Convection (Oxford: Oxford Univ. Press)

Erkaev, N. V., Kulikov, Y. N., Lammer, H., et al. 2007, A\&A, 472, 329

Feistel, R., \& Wagner, W. J. 2006, Phys. Chem. Ref. Data, 35, 1021

French, M., Mattsson, T. R., Nettelmann., N., \& Redmer, R. 2009, Phys. Rev. B, 79, 054107

García Muñoz, A. 2007, Planet. Space Sci., 55, 1426

Goncharov, A. F., et al. 2009, J. Chem. Phys., 130, 124514

Guillot, T. 2005, Annual Review of Earth and Planetary Sciences, 33, 493

Guillot, T., Gautier, D., Chabrier, G., \& Mosser, B. 1994, Icarus, 112, 337
Guillot, T., \& Morel, P. 1995, A\&AS, 109, 109

Hemley, R. J., Jephcoat, A. P., Mao, H. K., et al. 1987, Nature, 330, 737

Hirschmann, M. M. 2000, Mantle solidus: Experimental constraints in the effects of peridotite composition. G-cubed 1

Hubbard, W. B., Pearl, J. C., Podolak, M., \& Stevenson, D. J. 1995, The Interior of Neptune, in Neptune and Triton, ed. D. P. Cruikshank (Tucson: Univ. of Arizona Press), 109

Ikoma, M., \& Genda, H. 2006, ApJ, 648, 696

Iro, N., Bézard, B., \& Guillot, T. 2005, A\&A, 436, 719

Kerley, G. I. 1972, Los Alamos Lab. Rep. LA-4776 (Los Alamos: LANL)

Lammer, H., et al. 2009, A\&A, 506, 399

Lecavelier des Etangs, A., Vidal-Madjar, A., McConnell, J. C., \& Hébrard, G. 2004, A\&A, 418, L1

Leger, A., Rouan, D., Schneider, R., et al. 2009, A\&A, 506, 287

Lin, D. N. C., Bodenheimer, P., \& Richardson, D. C. 1996, Nature, 380, 606

Lunine, J. I., Hubbard, W. B., Burrows, A., Wang, Y.-P., \& Garlow, K. 1989, ApJ, 338, 314

Marley, M. S., Gómez, P., \& Podolak, M. 1995, J. Geophys. Res., 100, 23349

McDonough, W. F., \& Sun, S.-S. 1995, Chem. Geol., 120, 223

McNeil, D. S., \& Nelson, R. P. 2010, MNRAS, 401, 1691

Mibe, K., Kanzaki, M., Kawamoto, T., et al. 2007, J. Geophys. Res., 112, B03201

Moorhead, A. V., \& Adams, F. C. 2005, Icarus, 178, 517

Morard, G., Guyot, F., Bouchet, J., \& Mazevet, S. 2009, EPSL, submitted

Murray-Clay, R. A., Chiang, E. I., \& Murray, N. 2009, ApJ, 693, 23

Ohtani, E., \& Kamaya, N. 1992, GRL, 19, 2239

Presnall, D. C., 1994, Phase Diagram of Earth-Forming Minerals, in Mineral Physics and Crystallography A Handbook of Physical Constants, ed. T. J. Ahrens (AGU), 354

Queloz, D., Bouchy, F., Motou, C., et al. 2009, A\&A, 506, 303

Ribas, I., Guinan, E. F., Güdel, M., \& Audard, M. 2005, ApJ, 622, 680

Saumon, D., Chabrier, G., \& van Horn, H. M. 1995, ApJS, 99, 713

Schaefer, L., \& Fegley, B. 2007, Icarus, 186, 462

Schaefer, L., \& Fegley, B. 2009, ApJ, 703, L113

Schwager, B., Chudinovskikh, L., Gavriliuk, A, \& Boehler, R. 2004, J. Phys.: Condens. Matter, 16, 1177

Shen, A. H., \& Keppler, H. 1997, Nature, 385, 710

Stevenson, D. J. 2008, Nature, 451, 261

Tian, F., Kasting, J. F., Liu, H.-L., \& Roble, R. G. 2008, J. Geophys. Res. (Planets), 113, 5008

Tsuchiya, T., Jun, T., Kiochiro, U., \& Renata, M. W. 2004, Earth Planet. Sci. Lett., 224, 241

Valencia, D., O'Connell, R. J., \& Sasselov, D. D. 2006, Icarus, 181, 545

Valencia, D., Sasselov, D. D., \& O'Connell, R. J. 2007a, ApJ, 656, 545

Valencia, D., Sasselov, D. D., \& O'Connell, R. J. 2007b, ApJ, 665, 1413

Verner, D. A., Ferland, G. J., Korista, K. T., \& Yakovlev, D. G. 1996, ApJ, 465, 487

Vidal-Madjar, A., Lecavelier des Etangs, A., Désert, J.-M., et al. 2003, Nature, 422,143

Vidal-Madjar, A., Désert, J.-M., Lecavelier des Etangs, A., et al. 2004, ApJ, 604, L69

Wagner, W., \& Pruß, A. 2002, J. Phys. Chem. Ref. Data, 31, 387

Watson, A. J., Donahue, T. M., \& Walker, J. C. G. 1981, Icarus, 48, 150

Wu, Y., \& Murray, N. 2003, ApJ, 589, 605

Yelle, R., Lammer, H., \& Ip, W.-H. 2008, Space Sci. Rev., 139, 437 